\title{
Damage Progression in Compressively Loaded Laminates Containing a Circular Cutout
}

\author{
A. M. Waas* \\ University of Michigan, Ann Arbor, Michigan 48109 \\ and \\ C. D. Babcock Jr. $\dagger$ and W. G. Knauss $\ddagger$ \\ California Institute of Technology, Pasadena, California 91125
}

\begin{abstract}
A series of experiments have been performed to determine the mechanisms of failure in compressively loaded laminated plates in the presence of stress gradients generated by a circular cutout. Real time holographic interferometry and in situ photomicrography of the hole surface along with strain gauge measurements are used to study the progression of failure. The test specimens are multilayered composite flat plates that are loaded in compression. The laminates are made up of two material systems, T300/BP907 and IM7/8551-7. Two different lay-ups of both systems are investigated. The experimental results reveal that the failure is initiated as a localized instability in the 0 deg plies at the hole surface approximately at right angle to the loading direction. Subsequent to the 0 deg ply failure, extensive delamination cracking is observed with increasing load. The delaminated portions spread to the undamaged areas of the laminate by a combination of delamination buckling and growth, the buckling further enhancing the growth.
\end{abstract}

\section{Introduction}

$\mathbf{T}$ HE use of graphite/epoxy laminates in aerospace engineering applications is abundant. These laminates offer an attractive combination of high strength and low density over their traditional metal counterparts. In many instances, it is necessary to adopt techniques that are not conventional in characterizing these materials and that differ from isotropic and homogeneous material in their response to mechanical load.

A considerable amount of research effort has been expended in attempting to understand the failure processes of composite laminates, especially in a compressive load environment. Such a task is made difficult by the several competing modes of failure that are prevalent in a given situation. From a design standpoint, the researcher needs to identify the most important aspect of the failure process. When structural components are made out of these laminates, it is seldom that they will persist in an environment free of stress concentrations. Stress raisers can be lethal and are potential sites where damage can initiate in a structure. In the present work, we shall be concerned with the failure processes in fibrous composite laminates in the presence of stress concentrations.

The open hole strengths of these laminates has been the subject of numerous investigations. ${ }^{1-4}$ Figure 1 depicts the ultimate load carrying capacity of these laminates as a function of the normalized hole diameter. Mikulas ${ }^{5}$ has shown that these results could be bounded by simple criteria based on notch sensitivity. If the material is notch insensitive, the failure strains are directly proportional to the reduction in crosssectional area, whereas if the material is notch sensitive, then it is postulated that the material fails when the strain concen-

Presented as Paper 89-1274 at AIAA 30th Structures, Structural Dynamics and Materials Conference, April 3-5, 1989; received July 11, 1989; revision received Jan. 30, 1990. Copyright (C) 1990 by the American Institute of Aeronautics and Astronautics, Inc. All rights reserved.

*Assistant Professor, Department of Aerospace Engineering. Member AIAA.

†Deceased; formerly, Professor of Aeronautics and Applied Mechanics.

$\ddagger$ Professor of Aeronautics and Applied Mechanics. tration at the hole edge equals the failure strain of the material. It is seen that the latter assumption is too conservative in that the failure strain is underestimated for $a / w$ ratios $<0.4$. This situation indicates the possibility that the strain concentration effect alone cannot possibly account for the failure process. Based on this premise, Starnes and Williams ${ }^{l}$ carried out open hole strength testing of these laminates and reported a shear crippling type of failure to be prevalent in the vicinity of the hole prior to catastrophic failure. This conclusion was based on postmortem examination of damaged specimens.

The progressive nature of failure, in many instances by various stages, is a characteristic property of laminated composites. Several different failure processes are prevalent when these laminates are stressed, and what is required is to identify the key aspects of failure in a particular situation.

The present paper, which is condensed from Ref. 6, describes a detailed experimental investigation to understand the initiation and propagation of failure in laminates in the presence of a stress raiser. A companion paper ${ }^{17}$ contains certain other aspects that are relevant to the present topic and, thus, some repetition is unavoidable.

The stress concentration effect is produced by choosing a flat plate with a centered circular hole and subjecting it to an

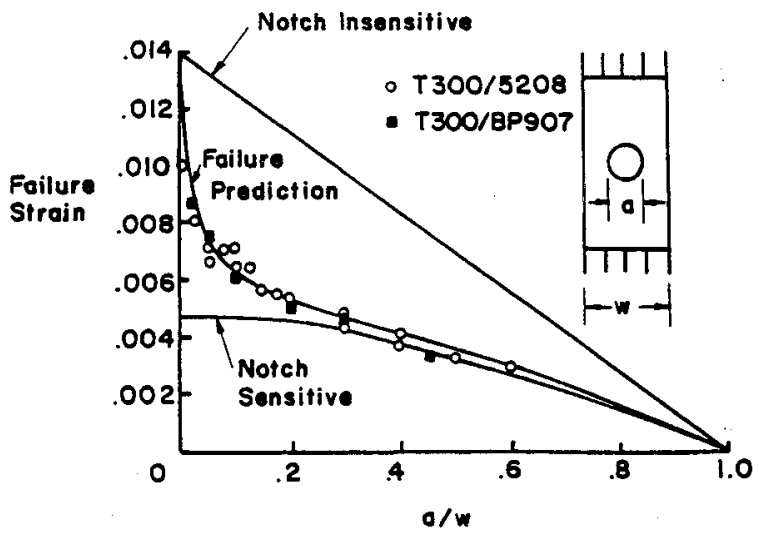

Fig. 1 Summary of test results for T300/BP907 and T300/5208 composite laminates: $w=13 \mathrm{~cm}$ (from Ref. 1) 
in-plane compressive load. The hole is carefully cut in order to insure that excessive local damage does not occur during the machining process. The damage initiation and propagation throughout the entire load history is studied via real time holographic interferometry and photomicrography of the hole surface. Strain gauge measurements are carried out on the IM7/8551-7 specimens. The results thus obtained are substantiated by postexperiment examination of the damaged specimens ultrasonically and under an optical microscope.

\section{Experiment}

\section{A. Assembly}

Figure 2 is a photograph showing the arrangement of the various devices used in the experiment. On a pneumatically vibration isolated table, optical components are arranged to make a hologram and generate interferograms of the test specimen, which is loaded by a table top compression device. An optical microscope is mounted on a tripod that is situated adjacent to the optical table by the testing machine. A $35-\mathrm{mm}$ motor driven single lens reflex camera attached to the microscope is used to take photographs of the test specimen hole surface during the testing sequence. Recording and developing of the hologram is done on a thermoplastic plate that is housed in the body of the instant recording device (IRD), as marked in Fig. 2. This device, which is electronically controlled, replaces the conventional wet chemical processes that are used if the recording is done on a photographic plate. A video camera placed behind the thermoplastic plate views the test specimen through the hologram. A VCR records the interferometric pattern generated during the loading sequence. A television monitor is used to view the interferograms during a typical test, which enables testing to be done interactively. In the following sections, the main components of the experiment are described.

\section{B. Specimens}

The multilayered laminated plates used in the tests were supplied by NASA Langley Research Center. The composition of the laminae in the specimen consisted of Thornel-300 graphite fibers preimpregnated with American Cyanamide BP907 epoxy resin and IM7 graphite fibers preimpregnated in 8551-7 epoxy resin. The IM7 fibers have a smaller diameter $(5 \mu \mathrm{m})$ than the T300 fibers $(7 \mu \mathrm{m})$. The fiber volume fraction in both these systems is $0.5-0.6$. These laminates are typical of those used in the aerospace industry. Two types of lamina stacking sequence were used in the tests. The first, designated type $\mathrm{A}$, is 48 ply $[+45 /-45 / 0 / 0 /+45 /-45 / 0 / 0 /+45 /-45 / 0 / 90]_{2 s}$, whereas the other, type $B$, is obtained by rotating the first sequence by $90 \mathrm{deg}$, the angles being measured with respect to the loading direction counterclockwise. Two sizes of specimen are used. The first, designated $\mathrm{S}$, is $7.62 \times 10.2 \mathrm{~cm}$ and the other, $\mathrm{L}$ is $12.7 \times 15.3 \mathrm{~cm}$. Hole sizes of $2.54,1.90$, and 1.27

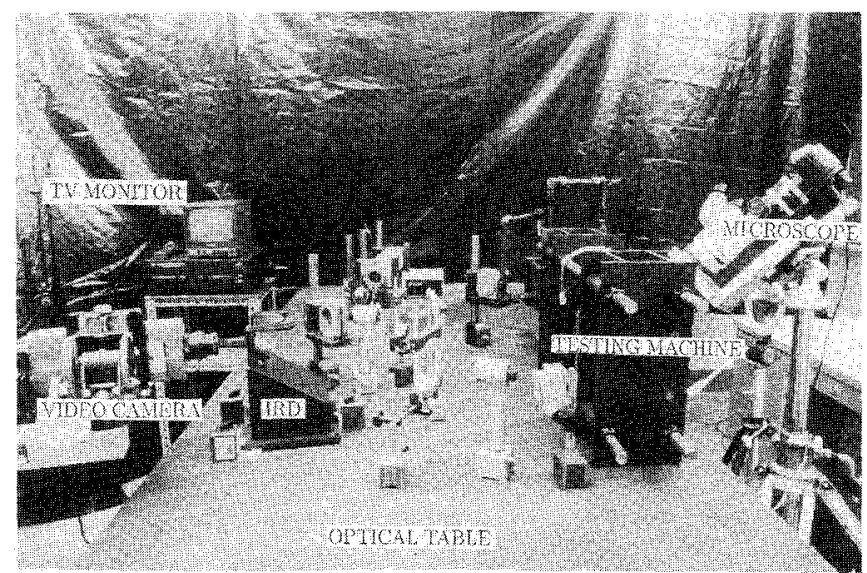

Fig. 2 Experimental setup. $\mathrm{cm}$ are used. For ease of presentation, the specimens are referred to as groups AS, BS, AL, and BL. Thus, AS, for example, refers to type A, small.

\section{Holographic Interferometry}

Real time holographic interferometry is used in this study to record the out-of-plane displacement changes $\Delta w$ of the specimen during the loading sequence. The relevant details of the technique as applicable to the present experiment are given in Sec. E. One novelty in the present experiment is the use of an electronically controlled process in the recording and development of a hologram. In conventional holography, a wet chemical process is used in the development of the photographic glass plates. The time consumed during this process, which is of the order of 7-9 min, is a significant portion of the total duration of a test that is of the order of $2 \mathrm{~h}$. During a continuous loading sequence, the information on the displacement history is lost during this development time. The electronic process referred to earlier and explained in the next section overcomes this drawback.

\section{Instant Recording Device}

In this reconstruction process of a hologram, the conventional silver halide photographic plates cause diffraction via an amplitude grating that is representative of the spatial distribution of light (amplitude and phase) that is emitted from the diffusely reflective surface of the object. However, the same task could be accomplished with a phase grating, i.e., a surface contour variation that is representative of the light off of the object. This is the basic idea behind the success of the thermoplastic recording device. The device has several stages of operation, as detailed in Ref. 8.

\section{E. Experimental Procedure}

A schematic of the holographic interferometer used in this study is shown in Fig. 3. It is standard off-axis holographic system. Proper choice of reference to object beam intensity ratio is important for producing the best holograms. With the use of the IRD, the quality of the hologram is insensitive to the exposure time. In the real time mode, the test specimen is illuminated in a state $S_{1}$, for example, with coherent light transmitted from the laser via the object wave path (this con-

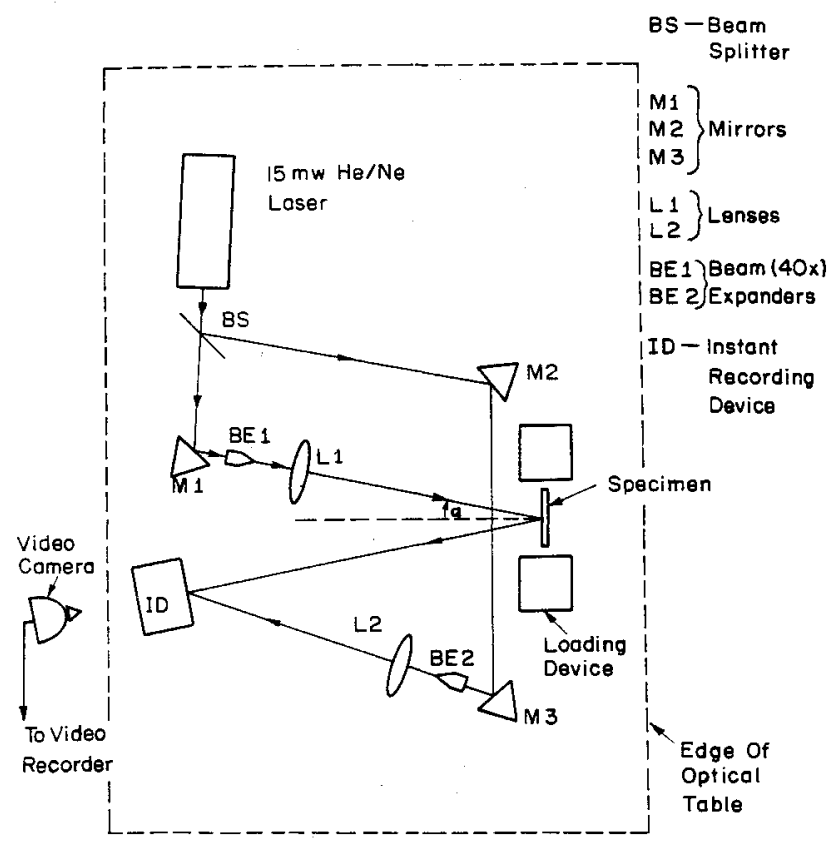




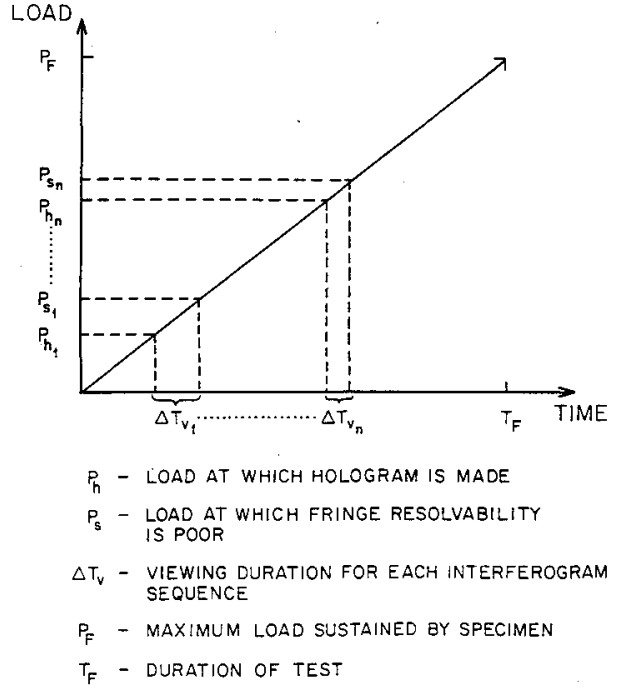

Fig. 4 Experimental procedure for generating interferograms.

sists of the beam splitter, a beam expander-spatial filter, and a collimating lens). The corresponding load is $P_{1}$ (refer to Fig. 4). A hologram is then recorded by exposing the thermoplastic plate positioned at the junction of the reflected light and reference beam. On completion of the first exposure, the specimen that is continuously loaded is viewed through a camera attached to a VHS recording system (a VCR and a monitor). The resulting image consists of fringes that are dynamical in nature representing contour maps of the changes, with respect to the state $S_{1}$, of the out of plane displacement component $w$ of the stressed specimen. These fringes, together with the load cell reading are recorded. The load cell reading is obtained by focusing the display of the voltmeter, exhibiting the load cell reading, at the position of the circular hole in the plane of the test specimen. With increasing load on the specimen, the fringe density becomes high, so that the resolution of the interferogram becomes poor. At this stage, a new hologram of the test specimen is made and the cycle of events is repeated. Thus, at the end of each experiment, we obtain an interferometric recording of the complete load vs out-of-plane displacement history of the specimen. This sequence of events is depicted in Fig. 4.

Each of the holographic interference fringe field is related to the change in surface displacement of the specimen under load by the vector expression

$$
n \lambda=\tilde{\boldsymbol{k}} \cdot \tilde{\boldsymbol{d}}
$$

where $n$ is the fringe number, $\lambda$ the wavelength of the coherent laser light, $\tilde{\boldsymbol{k}}$ the sensitivity vector $\left(\tilde{i_{2}}-\tilde{i_{1}}\right), \tilde{\boldsymbol{d}}$ the surface displacement vector at point of observation, and $\tilde{i_{1}}$ and $\tilde{i_{2}}$ the unit vectors in the illumination and observation directions, respectively. In the present experimental setup, the diffusely reflective surface of the specimen is oriented normal to the bisector of the angle $2 \alpha$, between $\tilde{i_{1}}$ and $\tilde{i_{2}}$ (Fig. 3). Thus, since the sensitivity vector $\tilde{\boldsymbol{k}}$ also points along this bisector, the interferometer senses only the out-of-plane displacement component $w$. Equation (1) then becomes

$$
\begin{aligned}
& n \lambda=2 w \cos \alpha \\
& w=n \lambda / 2 \cos \alpha
\end{aligned}
$$

In the present experimental setup, the surface normal is 10 deg off the observation direction. With $\lambda=0.633 \mu \mathrm{m}$, this shows that each fringe represents an out-of-plane displacement change of $0.321 \mu \mathrm{m}$.

During the entire loading program, the surface of the hole is viewed through a microscope at an oblique angle and photo-
Table 1 Experimental results for T300/BP907 laminates

\begin{tabular}{lcccc}
\hline \hline Specimen/type & $\begin{array}{c}\text { Hole diameter, } \\
\mathrm{cm}\end{array}$ & $\begin{array}{c}\text { Thickness, } \\
\mathrm{mm}\end{array}$ & $\boldsymbol{P}_{i}, \mathrm{kN}$ & $\boldsymbol{P}_{f}, \mathrm{kN}$ \\
\hline TB1-AS & 2.54 & 7.51 & 126.0 & $127.0^{\mathrm{a}}$ \\
TB2-AS & 2.54 & 7.51 & 124.0 & $125.0^{\mathrm{a}}$ \\
TB10-AS & 2.54 & 7.53 & 124.0 & $\mathbf{1 2 7 . 0}$ \\
TB12-AS & 2.54 & 7.53 & 125.0 & 127.0 \\
TB7-BS & 2.54 & 7.51 & 64.0 & 91.0 \\
TB13-BS & 2.54 & 7.47 & 69.0 & 90.0 \\
TB6-BL & 2.54 & 7.51 & 127.0 & 168.0 \\
TB11-BL & 2.54 & 7.53 & 123.0 & $165.0^{\mathrm{b}}$ \\
TB9-BL & 2.54 & 7.51 & 119.0 & 169.0 \\
TB14-AS & 1.90 & 7.47 & 130.0 & 142.0 \\
TB17-AS & 1.90 & 7.53 & 128.0 & $143.0^{\mathrm{b}}$ \\
TB16-BS & 1.90 & 7.47 & 70.0 & $97.0^{\mathrm{b}}$ \\
TB18-BS & 1.90 & 7.53 & 79.0 & 110.0 \\
TB15-AS & 1.27 & 7.47 & 152.0 & $161.0^{\mathrm{b}}$ \\
\hline \hline a & & &
\end{tabular}

unloaded for microscopic examination.

Table 2 Experimental results for IM7/8551)-7 laminates

\begin{tabular}{lcccc}
\hline \hline Specimen/type & $\begin{array}{c}\text { Hole diameter, } \\
\text { cm }\end{array}$ & $\begin{array}{c}\text { Thickness, } \\
\text { mm }\end{array}$ & $P_{i}, \mathrm{kN}$ & $P_{f}, \mathrm{kN}$ \\
\hline IM2-AS & 2.54 & 7.44 & 128.5 & 144.1 \\
IM4-AS & 2.54 & 7.31 & 135.7 & 141.9 \\
IM5-AS & 2.54 & 7.33 & 124.8 & 129.0 \\
IM7-AS & 2.54 & 7.33 & 125.0 & 133.2 \\
IM16-AS & 2.54 & 7.32 & 119.2 & $128.3^{\mathrm{a}}$ \\
IM20-AS & 2.54 & 7.36 & 127.7 & 132.8 \\
IM21-AS & 2.54 & 7.32 & 125.0 & $134.3^{\mathrm{a}}$ \\
IM29-AS & 2.54 & 7.32 & 125.9 & 133.2 \\
IM1-BS & 2.54 & 7.38 & 68.7 & 97.8 \\
IM3-BS & 2.54 & 7.32 & 72.0 & 88.3 \\
IM6-BS & 2.54 & 7.36 & 70.5 & 92.1 \\
IM8-BS & 2.54 & 7.36 & 67.2 & $89.4^{\mathrm{a}}$ \\
IM9-BS & 2.54 & 7.33 & 72.1 & $89.8^{\mathrm{a}}$ \\
IM19-BS & 2.54 & 7.36 & 68.9 & $76.3^{\mathrm{a}}$ \\
IM32-BS & 2.54 & 7.30 & 60.0 & 90.3 \\
IM24-BL & 2.54 & 7.36 & 127.0 & $136.3^{\mathrm{a}}$ \\
IM25-BL & 2.54 & 7.44 & 123.2 & $165.5^{\mathrm{a}}$ \\
IM28-BL & 2.54 & 7.33 & 114.3 & 164.1 \\
\hline \hline
\end{tabular}

${ }^{\mathrm{a}}$ Maximum load sustained. Specimen unloaded for microscopic examination.

graphs are taken at various times. Because of the oblique viewing angle, the hole surface appears curved on the photomicrographs. A fiber optic cable attached to a high-intensity lamp is used to illuminate the hole surface. The interferograms displayed on the TV monitor helps in taking these photomicrographs interactively in an efficient manner.

The interferometric data reveal the changes in the $w$ displacement component. These changes may be the manifestations of internal damage. Thus, some specimen were loaded to a post initiation level but were unloaded prior to catastrophic failure. The unloaded specimens were sectioned in the region of stress concentration and were examined under an optical microscope. In the following section, the results obtained from interferometry, photomicrography, and interior damage studies are presented.

\section{Results and Discussion}

Tables 1 and 2 summarize the test conditions and the results obtained. In these tables, AS, for example, refers to type A, small. $P_{i}$ is the load at which failure initiates, as detected by the interferometer. $P_{f}$ is the failure load of the specimen. The interferometric data are presented only in the immediate neighborhood of the hole. The photomicrographs are only captured on one side of the hole surface. The horizontal shiny stripes appearing on the photomicrographs are 0 deg plies and vertical lines are pencil marks for purposes of reference.

Consider now the different stages of failure as exhibited by the interferometric results and substantiated by the photomicrographs. In the predamaged state, an increase in the load on the specimen results in a uniform pattern of fringes. These fringes are few in number and correspond to the thickness 
changes due to the Poisson's ratio effect, but coupled with some rigid-body motion and/or slight bending. The rigidbody motion and the bending cannot be avoided totally in a compression test. The slight bending may be the result of any initial imperfection in the specimen. Added to this, one must realize the high sensitivity of the measurement technique, which is a function of the wavelength of the laser light (displacement changes as small as $0.321 \mu \mathrm{m}$ can be detected). In the discussion to follow, the T300/BP907 material system will be referred to as TB, and the IM7/8551-7 will be referred to as IM.

Figure 5 shows a series of interferometric patterns of a type B specimen that correspond to the first instance at which internal damage was detected. In these interferograms, the axis of loading is horizontal. The numbers below each picture represent the load in kilo Newtons $\left[\left(4.448 \times 10^{3}\right)\right]^{-1}$ lbf corresponding to each frame, whereas $P_{h}$ indicates the load at which the hologram was made for that particular set of frames (reference load). Notice the highly localized fringe cluster adjacent to the hole edge at approximately $90 \mathrm{deg}$ to the loading direction. When any form of internal damage occurs that manifests itself as a localized perturbation in the normal displacement component $w$, the resulting interferometric patterns display this event as shown in this figure. A view of the hole surface corresponding to this fringe pattern is shown in frame 2 of Fig. 6. Notice the damage to the midplane 0 deg plies, which appear as a black spot. Adjacent to this spot is a delamination crack. Inspection of the photomicrographs prior to this event revealed no damage to the hole surface. In all of the experiments conducted, it was always revealed that a perturbation in the interferometric data corresponded to 0 deg ply damage at the hole surface. This interferometric pattern is interpreted as a localized bulging of the surface just above the damage site. This localized damage to the 0 deg plies is found to be the triggering of extensive delamination cracking and

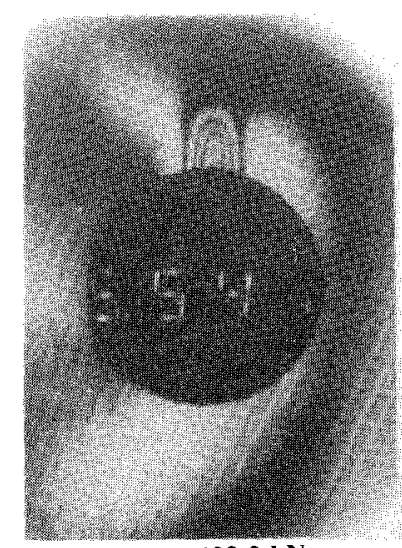

123.2 kN

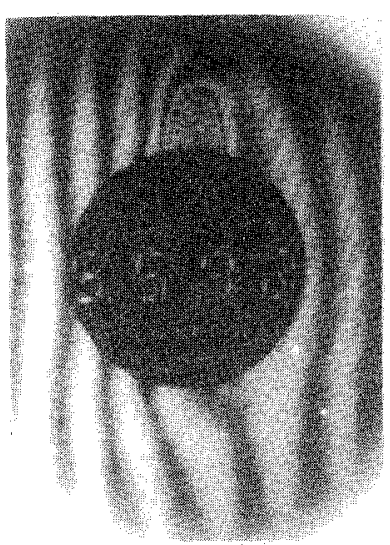

$124.4 \mathrm{kN}$

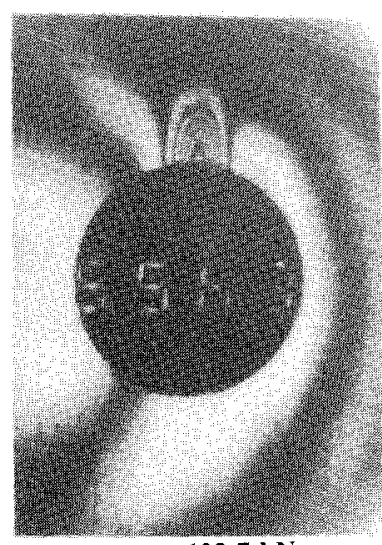

$123.7 \mathrm{kN}$

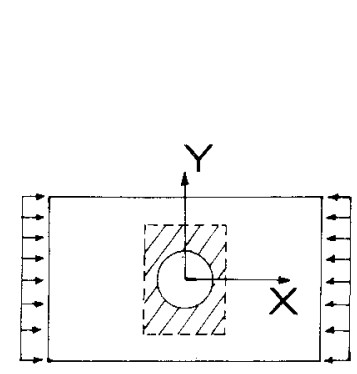

$P_{h}=123.2$
Fig. 5 Initiation of damage (specimen TB11). localized buckling of the delaminated portions that follows subsequently. This sequence of events is observed consistently in all of the type B specimen. At this stage, it is pointed out that the word damage is used to describe any form of destruction to the specimen caused by the application of mechanical load as picked up on the interferograms. The interferograms corresponding to the initiation event reveal the displacements within the damage zone to be increasing with increasing load. This is clear by noting a sharp increase in the fringe density within the damage zone as loading proceeds. Subsequent to the initiation, the remaining $0 \mathrm{deg}$ plies undergo failure while delamination are seen to appear between various ply interfaces. An inspection of the sequence of photomicrographs in Fig. 6 shows this. The delamination undergo a stage of slow growth, as shown in the next set of interferometric patterns (Fig. 7). Here, the reference state corresponds to a load $P_{h}=$ $159 \mathrm{kN}$ while the three frames span a load increase $\Delta P$ of 1.73 $\mathrm{kN}$. The displacement patterns extracted from the interferograms in Fig. 7 are shown in Fig. 8. Here, the displacements are measured with respect to displacements in areas remote from the hole edge. The origin of the axes is placed at the edge of the hole as indicated. The $X$ and $Y$ axes are scaled, whereas the vertical $Z$ axes, representing $\Delta w$, is $1: 1$. The fringe patterns in Fig. 7 reveal the displacements to be growing faster within the damage zone than the rest of the specimen. Further, the damage area is also seen to grow, indicating that the delaminated portions are spreading outward approximately at right angles to the direction of the applied load. The corresponding photomicrographs (Fig. 6) show the extent to which delamination cracking occurs. Notice the zero fiber breaks as well as the large (on the order of ply thickness) mode I type displacements. These observations lead us to conclude that delamination buckling is the mechanism chiefly responsible for the spreading of the damage to the undamaged areas of the plate. Once the delaminated portions reach a sizable extent, an increase in the growth rate is noticed. This is shown in the last series of interferograms (Fig. 9). In these, the fringe density within the damage zone is very high, so much so that the resolution of the interferogram within this area is poor. However, the manner in which these delaminations grow and respond

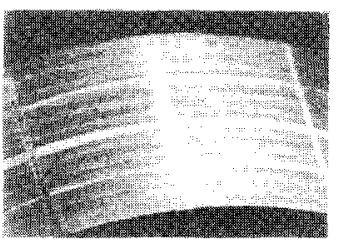

$66.7 \mathrm{kN}$

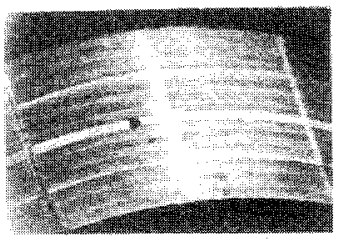

$123.2 \mathrm{kN}$
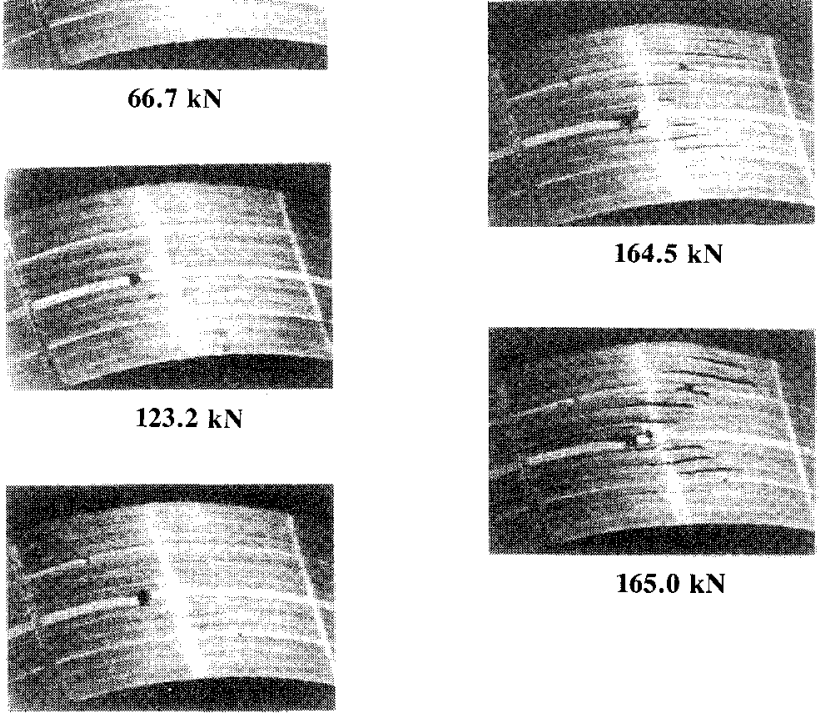

$164.5 \mathrm{kN}$

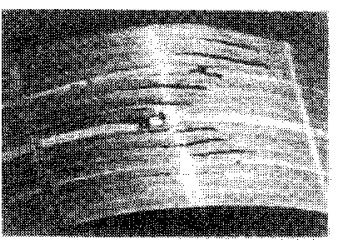

$165.0 \mathrm{kN}$
$159.1 \mathrm{kN}$

Fig. 6 Hole surface photomicrographs of specimen TB11 (type B). 
can be deduced by following the fringe development within the damage area. It must be pointed out that playback of the recorded video information displays a continuous transition in the fringe data, which enables the uninterrupted following of the damage progression as opposed to the limited number of frames displayed here. The present discussion is influenced from such knowledge. The culmination of the last growth event is the complete loss of flexural stiffness of each of the delaminated portions, leading to catastrophic failure. These

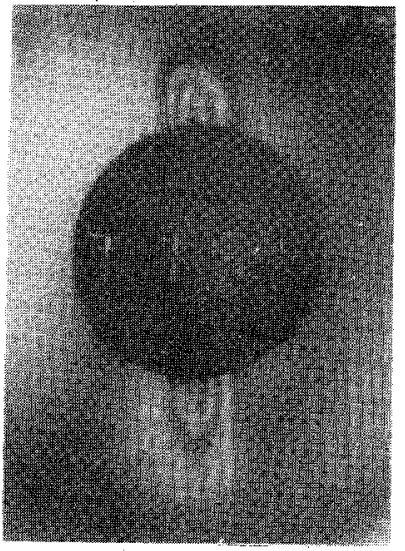

$159.1 \mathrm{kN}$

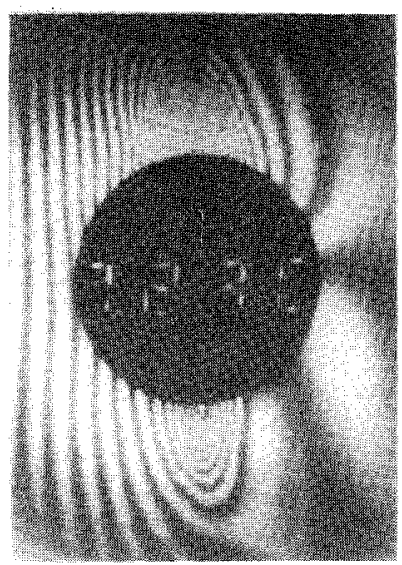

$160.8 \mathrm{kN}$

Fig. 7 Buckling and spreading of delaminated portions (specimen TB11).

$$
P_{h}=159.0
$$

findings and conclusions are drawn from the consistency with which these failure events occur in all type B specimen.

In a type A specimen ( $42 \% 0$ deg plies as contrasted with $8 \%$ in type B), the mechanisms leading to failure initiation and propagation are as discussed before for type B specimen, apart from the level of applied load at which the different events take place. This is evident from the results presented in Tables 1 and 2. A sharp localized cluster of fringes is seen to form at the edge of the hole, which spreads very rapidly with a small increase in applied load. The area in which the fringes are unresolvable extends out to about $80 \%$ of the hole radius from the hole edge. A corresponding set of photomicrographs, typical of these specimens, is shown in Fig. 10. Here, the initial 0 deg fiber failure occurs at $139 \mathrm{kN}$. With increasing load, the remaining 0 deg plies undergo failure. Adjacent to some of the damaged 0 deg plies, delamination cracking is found to occur. Notice the damage to all 0 deg plies and the formation of surface buckles on both surfaces of the specimen. A picture of the

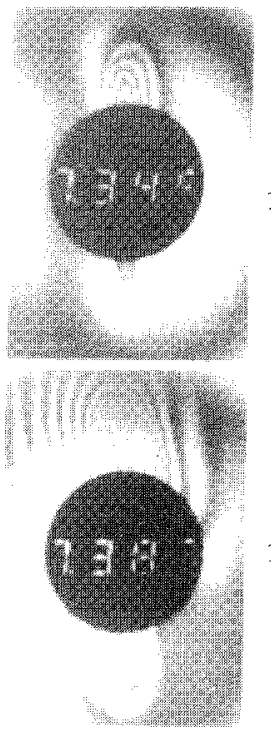

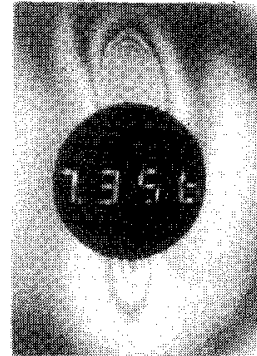

163.6

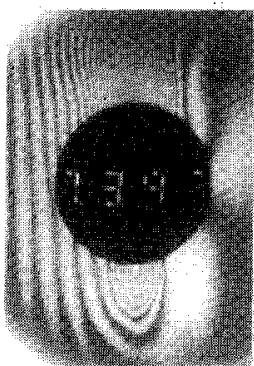

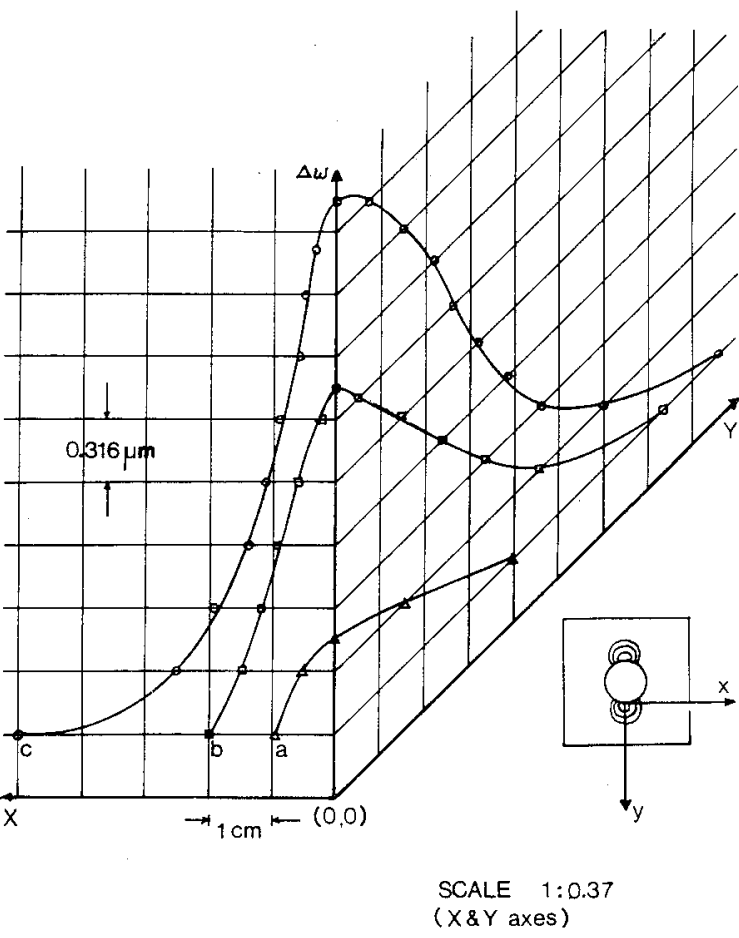

Fig. 8 Summary of displacements corresponding to Fig. 7.
163.4

$1.37 \mathrm{~cm}$

164.3

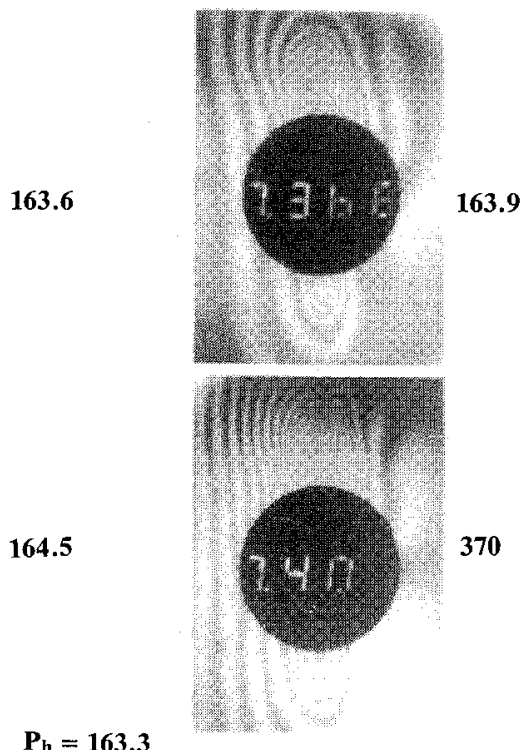

Fig. 9 Buckling and spreading of delaminated portions just prior to catastrophic failure (specimen TB11). 

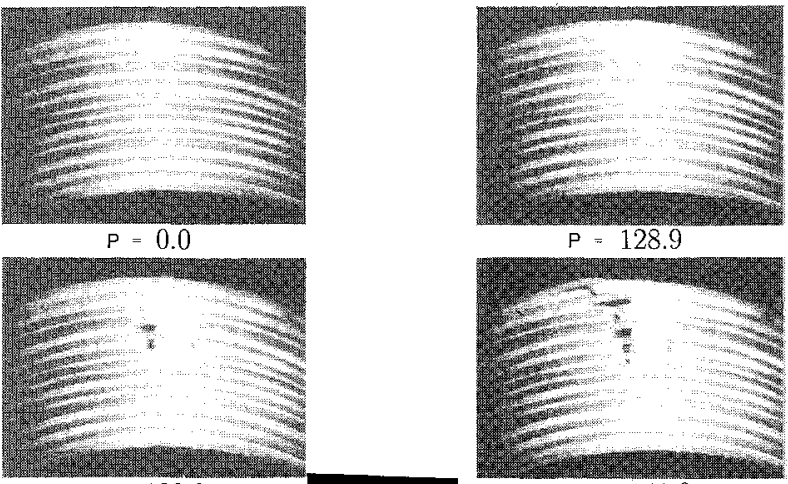

$P=139.0$
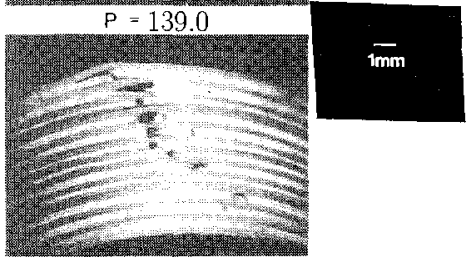

$P=142.3$

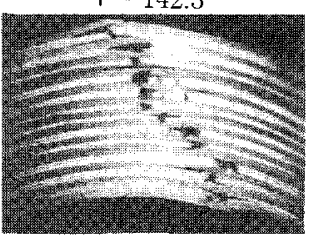

$\mathrm{P}=143.4$
$P=143.2$

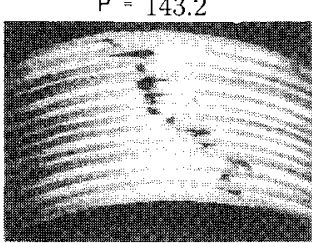

Unloaded

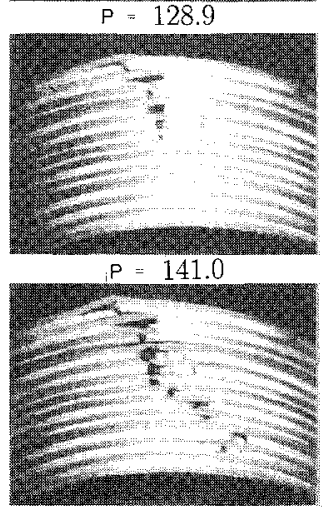

Fig. 10 Hole surface photomicrographs showing damage progression (specimen TB17).

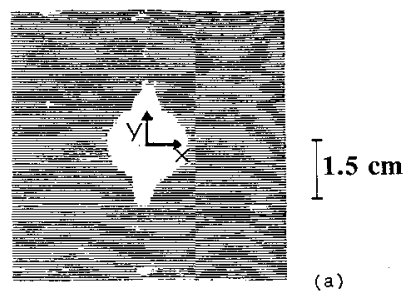

TB16

Fig. 11 C-scan map of typical damaged specimen (specimen TB16).

damaged, unloaded specimen is shown in the last frame of Fig. 10.

Bearing in mind the information obtained from the surfaces of the specimen, we now turn to a discussion of postexperiment sectioning studies. Shown in Fig. 11 is a typical C-scan map of a damaged specimen. In this figure, the extent of the damaged area is contained within about one hole radius. The damaged area is a function of the maximum load to which the specimen is loaded. Sectioning through the damaged area and observation under an optical microscope revealed several inclined narrow zones of fiber breaks within the 0 deg layers, identified as fiber kink bands. A typical section through the damaged area is shown in Fig. 12. Here, the interior 0 deg plies show several kinking failures. A typical complimentary kink band is shown in Fig. 12b. The geometry of a typical kink band is summarized in Fig. 13. We note in Fig. 12b a kink band inclination $\alpha$ of 65 deg to the load carrying direction. The lengths of the fibers in the inclined zone measures to 50-84 $\mu \mathrm{m}$. More important, it must be pointed out that the region between the inclined zone appeared undamaged and oriented parallel to the fiber direction. In a study by Evans and Adler, ${ }^{9}$ carbon fiber bundles of width $\approx 400 \mu \mathrm{m}$ oriented in three orthogonal directions were set in a graphite matrix and subjected to localized impact. Postexperimental examination revealed typical kink orientation angles $\alpha$ of $45-60$ deg and kink boundary orientation $\beta$ obeying $\beta \approx \alpha / 2$ for broad $(l / d \geq 0.1)$ kinks. Here $\beta$ is measured from the perpendicular to the fiber direction. For narrow kinks, $\beta$ was found to increase and $\alpha$ was random. Corresponding results in our study that fall into the category $l / d \geq 0.1$ indicate $\beta \approx 25-30 \mathrm{deg}$ and $\alpha \approx 15-30 \mathrm{deg}$. However, no definite trend between $\alpha$ and $\beta$ can be established. This type of kinking failure has also been observed in other studies dealing with compressive strengths of laminates (see, for example, Refs. 9-11).

\section{A. Strain Gauge Results}

It was desirable to obtain the local value of strain at which damage initiated. To do so, some IM series specimens were mounted with standard foil strain gauges. The strain gauges were connected to a Wheatstone bridge in a single active gauge configuration, and at each location back to back gauges were placed to obtain both the in-plane axial strain in the direction of applied load as well as the bending strain. The gauge out-
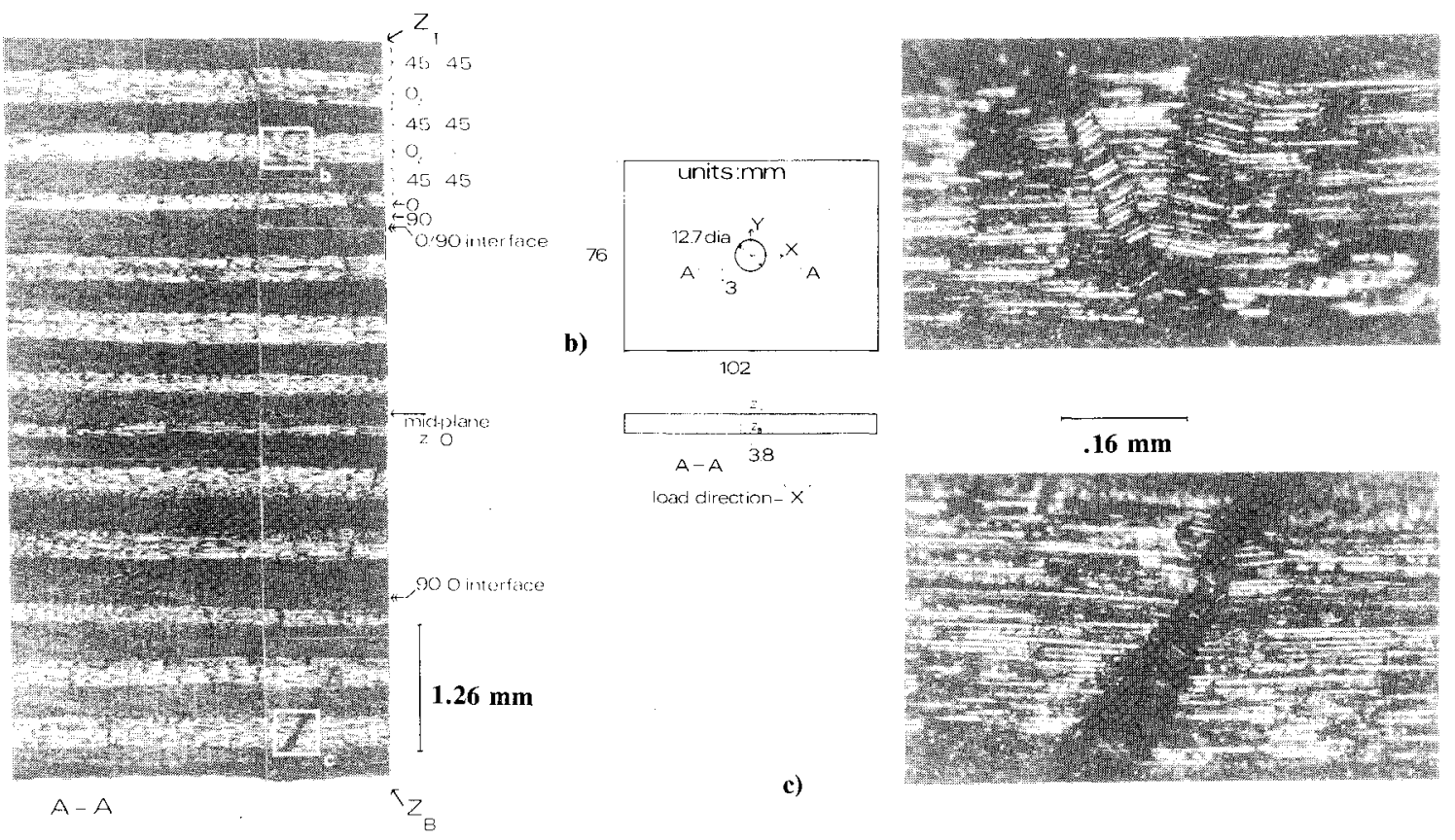

Fig. 12 Internal damage at the vicinity of the hole (specimen TB15): a) entire cross section; b) complementary kink in second 0 deg layer from top; and c) magnified view of damage to bottom $0 \mathrm{deg}$ layer. 
Table 3 Averaged local strain values at failure initiation

\begin{tabular}{cc}
\hline Specimen/type & Averaged strain, $\mu$ strain \\
\hline A & 8385 \\
B & 8612 \\
\hline
\end{tabular}
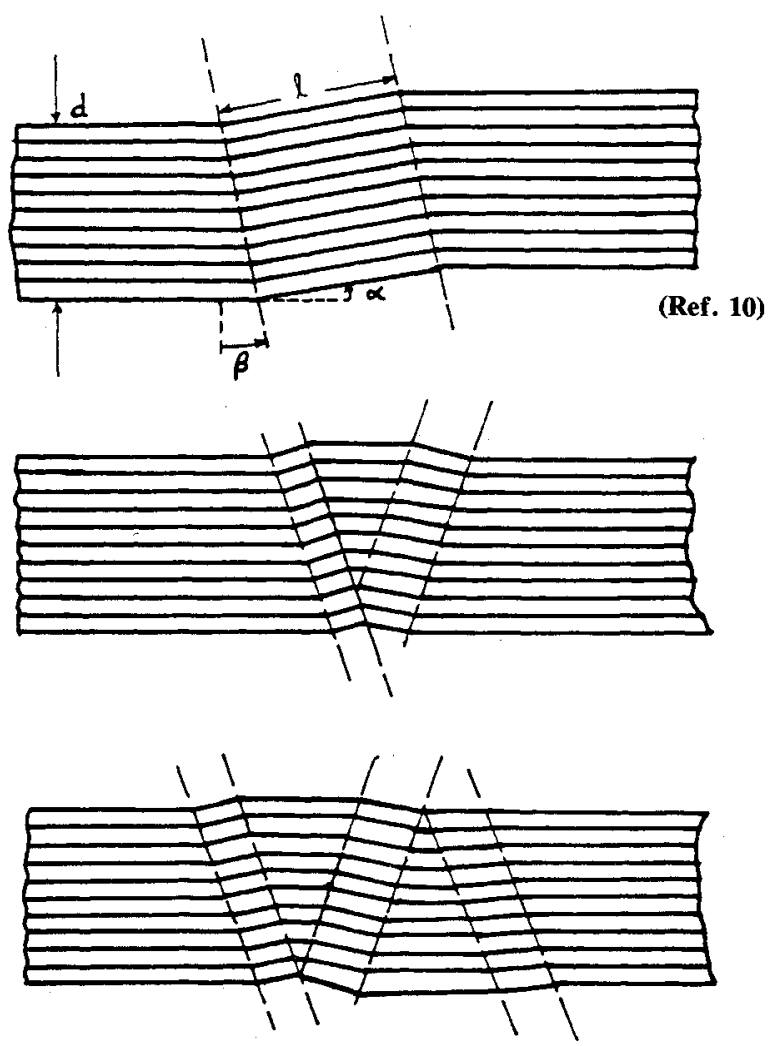

Fig. 13 Geometry of typical kink bands.

a)

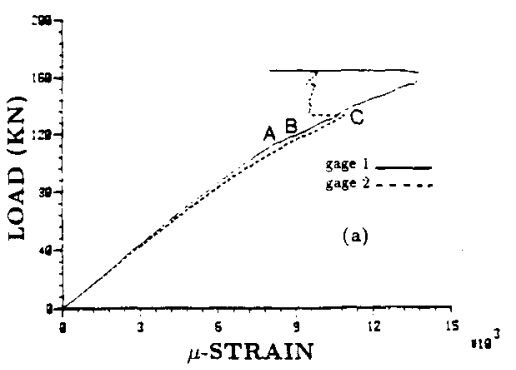

b)
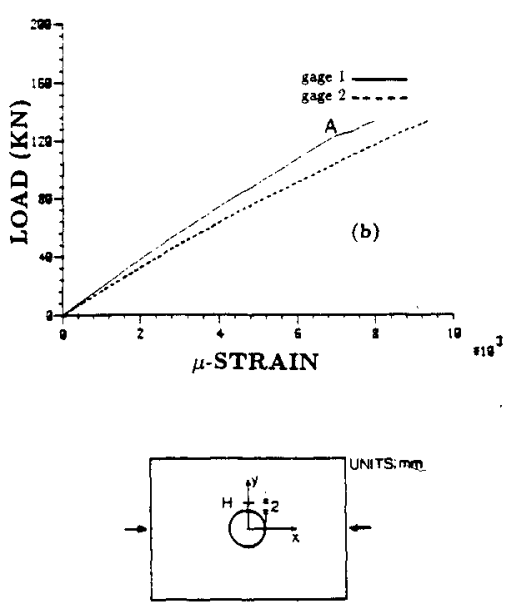

Fig. 14 Strain gauge response for specimens: a) type B (specimen IM28); and b) type A (specimen IM21). puts from the Wheatstone bridge were sent through an amplification stage and fed into an in-house computer for manipulation. Strain-load plots typical of type A and B specimens are shown in Fig. 14. In these figures, the response of the strain gauges, near the hole edge (location $\mathrm{H}$ ) is shown. Notice that the strains are seen to increase linearly with increasing far-field load. When the far-field load reaches $P_{i}$ (corresponding to damage initiation within the 0 deg plies), the load vs strain behavior shows a kink (marked A), implying a localized softening. For the type B specimen (Fig. 14a), with failure of other 0 deg plies, the softening continues (marked B), until reaching $C$, when strain reversal is detected on one gauge. This reversal occurs because the delaminated portions closet to the surface on which the gauge is attached undergo local buckling. The buckling results in tensile strains on the outer surface, due to bending, causing a net reduction in the compressive strain. For type A specimen, beyond failure initiation, data can be collected only to the point where surface buckles form due to delamination. At this time, the large out of plane displacements encountered during the rapid propagation and growth of the delaminated portion result in the strain gauges being peeled off of the surface, disrupting the data acquisition. The average microbuckling strain for the two types of specimens are listed in Table 3.

B. Summary of Common Features of Failure for Types $A$ and $B$.

In this section, we summarize the results presented so far and attempt to identify features that are common to types $\mathrm{A}$ and $\mathrm{B}$ laminates.

In both types $A$ and $B$, the failure is initiated by a 0 deg fiber failure at the hole edge approximately at right angles to the loading direction. In type $A$, with increasing load, more 0 deg fiber failure is seen to occur with simultaneous surface delamination buckling. These events occur very close to the catastrophic failure load, In type B, subsequent to 0 deg fiber failure that occurs at around $75 \%$ of the ultimate failure load, gradual development of delamination cracking is observed, with the outermost delaminated sections undergoing buckling. In both types, the delaminations are seen to grow with increasing loads. Delamination buckling is found to be the mechanism responsible for spreading of the damage. Finally, an accelerated growth of the delaminations is observed. At this time, the specimen fails catastrophically.

The mechanics of the $\mathbf{0}$ deg fiber failure, which shows up at the hole edge and is captured by the interferometer, is found to be a combination of localized fiber microbuckling/kinking. Information regarding fiber kinking published elsewhere and in here have been obtained once the damage has taken place and the structure loaded into a postdamaged state. Qualitative information regarding the predamaged state, from the interior of the structure, is difficult to obtain as these systems are opaque.

\section{Concluding Remarks}

In an attempt to understand the mechanisms for the initiation and spreading of damage in compressively loaded composite laminates containing a circular cutout, a detailed experimental investigation has been carried out. The investigation was aimed at recognizing the distinction between an unfailed laminate and a failed laminate and providing the transitional information that would serve to develop analysis capabilities with an underlying physical basis. This information is seldom obtained by following a purely theoretical path, and it was necessary that this problem be looked at experimentally. The following was found.

1) The initiation of failure in all of the laminate types investigated is by a combination of fiber microbuckling/kinking within the 0 deg plies. This failure gradually develops at the hole surface in the form of a fiber jutout failure. When the far-field load reaches a critical value, the corresponding localized value of strain at the hole edge reaches a critical value of 
$\approx 8500 \mu$ strain (deduced from the IM series specimen). At this value of local strain, fiber failure within the 0 deg plies is seen to occur, resulting in a propagation of the damage into the interior of the specimen. The damage zone extends out to approximately $75 \%$ of the hole radius. The microbuckling process results in the 0 deg fibers undergoing large rotation in plane $(X Y)$ as well as out of plane $(X Z)$. This leads to the formation of narrow $(l / d \approx 7-11)$ bands of broken fiber segments that align themselves at an inclination to the loading direction. The initiation occurs at a far-field applied load that is $\mathbf{7 5 - 9 5 \%}$ of the ultimate failure load of the specimen, the exact value depending on the laminate type. Thus, for all practical purposes, the laminate can be termed failed once failure of the 0 deg plies occurs.

2) The surface manifestation of the initiation process is captured by the interferometer as a well-defined perturbation in an otherwise smooth fringe pattern.

3) The interferometric data is substantiated by ultrasonic Cscanning, hole surface photomicrography, and sectioning studies under an optical microscope.

4) Subsequent to 0 deg ply damage, delamination cracks are seen to form at ply interfaces close to the location of the 0 deg plies as shown by the photomicrographs. The extent of the initial delaminated portion, the far-field load at which the delamination forms and the thickness of the delaminated portion, govern the occurrence of delamination buckling.

5) The buckling enhances growth of the delaminated portions that spread to the undamaged areas of the laminate approximately at right angles to the direction of the applied load.

6) When the delaminated area reaches a critical size, the growth process is seen to accelerate rapidly. The culmination of this last event is the catastrophic failure of the plate due to a complete loss of flexural stiffness of each of the delaminated portions. The load carrying capacity beyond initiation, at which stage delamination buckling/growth occurs, is dictated by the overall stiffness of the laminate, This in turn, is governed by the 0 deg ply percentage.

\section{Acknowledgment}

This research was supported by the NASA under Grant NSG-1483. The authors are appreciative of this support. The interest and encouragement of James H. Starnes, Jr., NASA Langley Research Center, in pursuing this work is gratefully acknowledged.

\section{Reference}

${ }^{1}$ Starnes, J., and Williams, J. G., "Failure Characteristics of Gr/Epoxy Structural Components Loaded in Compression," NASA TM 84552, 1982.

${ }^{2}$ Rhodes, M., Mikulas, M., and McGowan, P., "Effect of Orthotropic Properties and Panel Width on the Compression Strength of Gr/Epoxy Laminates with Holes," AIAA Paper 82-0749, 1982.

${ }^{3}$ Shuart M. J., and Williams J. G., "Compression Behavior of [+45/-45] Dominated Laminates with a Circular Hole or Impact Damage," AIAA Journal, Vol. 24, No. 1, pp. 115-122.

${ }^{4}$ Knauss, J., Starnes, J. and Henneke, E., "The Compressive Failure of Gr/Epoxy Plates with Circular Holes," NASA CR 157115, N78-24295, 1978.

${ }^{5}$ Mikulas, M., "Failure Prediction Techniques for Compression Loaded Composite Laminated," NASA CP 2142, 1980.

${ }^{6}$ Waas, A., "Compression Failure of Fibrous Laminated Composites in the Presence of Stress Gradients: Experiment and Analysis," Ph.D. Dissertation, California Inst. of Technology, Pasadena, CA, 1988.

${ }^{7}$ Waas, A., Babcock, C. D., and Knauss, W. G., "An Experimental Study of Compression Failure of Fibrous Laminated Composites in the Presence of Stress Gradients,"' International Journal of Solids and Structures, Vol. 26, No. 9-10, 1990, pp. 1071-1098.

${ }^{8}$ Owners Manual-HC300,301, Newport Research Corporation, CA, 1986.

${ }^{9}$ Evans, A., and Adler, A., "Kinking as a Mode of Structural Degradation in Carbon Fiber Composites," Acta Metallurgica, Vol. 26, 1977, pp. 725-738.

${ }^{10}$ Chaplin, C. R., "Compressive Fracture in Uni-Directional Glass Reinforced Plastics," Journal of Material Science, Vol. 12, 1977, pp. 347-352.

${ }^{11} \mathrm{Hahn}, \mathrm{H}$, and Williams, J. G., "Compression Failure Mechanisms in Unidirectional Composites," NASA TM 85834, 1984. 